\title{
A Prolog Simulator for Deterministic P Systems with Active Membranes
}

\author{
A. CORDÓN-FRANCO, M.A. GUTIÉRREZ-NARANJO, \\ M.J. PÉREZ-JIMÉNEZ, F. SANCHO-CAPARRINI \\ Dpto. de Ciencias de la Computación e Inteligencia Artificial \\ E.T.S. Ingeniería Informática. Universidad de Sevilla \\ Avda. Reina Mercedes s/n, 41012, Sevilla, España \\ \{acordon, magutier, marper, fsancho\}@us.es
}

Received 8 April 2003

\begin{abstract}
In this paper we propose a new way to represent P systems with active membranes based on Logic Programming techniques. This representation allows us to express the set of rules and the configuration of the $\mathrm{P}$ system in each step of the evolution as literals of an appropriate language of first order logic. We provide a Prolog program to simulate the evolution of these $\mathrm{P}$ systems and present some auxiliary tools to simulate the evolution of a $\mathrm{P}$ system with active membranes using 2-division which solves the SAT problem following the techniques presented in ${ }^{10)}$.
\end{abstract}

Keywords Logic programming, Membrane computing, Simulation, Prolog, SAT-problem

\section{$\S 1$ Introduction}

In ${ }^{5)}$, a new model of computation within the framework of Natural Computing was introduced, called P Systems.

It is based upon the notion of membrane structure that is used to enclose computing cells in order to make them independent computing units. Also, a membrane serves as a communication channel between a given cell and other cells "adjacent" to it. This model comes from the observation that the processes 
which take place in the complex structure of a living cell can be considered as computations.

Since these computing devices were introduced several variants have been considered. See ${ }^{6)}$ for a fairly complete compendium about $\mathrm{P}$ systems.

The different variants of $\mathrm{P}$ systems found in the literature are generally thought as generating devices. Many of them have been proved to be computationally complete ${ }^{6)}$.

The model we study here, $\mathrm{P}$ systems with active membranes, works with symbol-objects, and it provides rules for membrane division. In particular, $P$ systems with active membranes are studied in ${ }^{6}$, section 7.2.

The main goal of this paper is to propose and illustrate a representation of $\mathrm{P}$ systems with active membranes based on Logic Programming techniques.

The paper is organized as follows: Section 2 briefly presents some ideas about the convenience of using Prolog as the basis for this representation; Section 3 introduces the way to represent all basic ingredients of this model; Section 4 studies the designed algorithm to simulate deterministic $\mathrm{P}$ systems with active membranes; Section 5 presents as an example the solution given to SAT problem using this model in ${ }^{10)}$; Section 6 presents some conclusions and future work about the possibilities of this new simulator and representation techniques using Logic Programming; finally, the Appendix shows a standard work session with the interface provided with the simulator.

\section{$\S 2$ Why Prolog?}

Choosing a programming language for an effective implementation of a $\mathrm{P}$ system simulator is not an easy decision. The language has to be expressive enough to handle symbolic knowledge in a natural way and the ability of evolving the different configurations following a set of rules. Prolog ${ }^{* 1}$ has both these features.

On one hand, the based-tree data structure and the use of infix operators defined ad hoc by the programmer allow us to simulate the natural language and the user can follow the evolution of the system without any knowledge of Prolog (see sections 3.2 and 3.3). On the other hand, Prolog programs are sets of facts and rules and basic mechanisms of Prolog are pattern matching and automatic backtracking, so the design of the inference engine to perform the evolutions has a natural treatment from a programmer point of view.

\footnotetext{
${ }^{* 1}$ A good starting point can be ${ }^{3)}$ or ${ }^{13)}$.
} 
Finally, the use of Prolog as programming language has other desirable side effects which are out of the scope of this paper. Prolog fits into all kinds of symbolic reasoning and the use of this representation can be a way to link $\mathrm{P}$ systems to other deeply studied fields in Artificial Intelligence.

\section{$\S 3$ A Logic Programming representation of $\mathbf{P}$ systems with active membrane}

Following ${ }^{6)}$ a $P$ system with active membranes is a construct:

$$
\Pi=\left(V, H, \mu, w_{1}, \ldots, w_{m}, R\right),
$$

where:

1. $m \geq 1$, is the initial degree of the system;

2. $\quad V$ is the alphabet of symbol-objects;

3. $\quad H$ is a finite set of labels for membranes;

4. $\mu$ is a membrane structure, of $m$ membranes, labelled (not necessarily in a one-to-one manner) with elements of $H$;

5. $w_{1}, \ldots, w_{m}$ are strings over $V$, describing the initial multisets of objects placed in the $m$ regions of $\mu$;

6. $R$ is a finite set of evolution rules, of the following forms:

a. $\left[{ }_{h} x \rightarrow u\right]_{h}^{\alpha}$, for $h \in H, \alpha \in\{+,-, 0\}, x \in V, u \in V^{*}$. This is an object evolution rule, associated with a membrane labelled with $h$ and depending on the polarity of that membrane, but not directly involving the membrane.

b. $\quad x\left[{ }_{h}\right]_{h}^{\alpha_{1}} \rightarrow\left[{ }_{h} y\right]_{h}^{\alpha_{2}}$, for $h \in H, \alpha_{1}, \alpha_{2} \in\{+,-, 0\}, x, y \in V$. An object from the region immediately outside a membrane labelled with $h$ is introduced in this membrane, possibly transformed into another object, and simultaneously its polarity can be changed.

c. $\left[{ }_{h} x\right]_{h}^{\alpha_{1}} \rightarrow\left[{ }_{h}\right]_{h}^{\alpha_{2}} y$, for $h \in H, \alpha_{1}, \alpha_{2} \in\{+,-, 0\}, x, y \in V$. An object is sent out from membrane labelled with $h$ to the region immediately outside, possibly transformed into another object, and simultaneously the polarity of the membrane can be changed.

d. $\quad\left[{ }_{h} x\right]_{h}^{\alpha} \rightarrow y$, for $h \in H, \alpha \in\{+,-, 0\}, x, y \in V$. A membrane labelled with $h$ is dissolved in reaction with an object. The skin is never dissolved.

e. $\left[{ }_{h} x\right]_{h}^{\alpha_{1}} \rightarrow\left[{ }_{h} y\right]_{h}^{\alpha_{2}}\left[{ }_{h} z\right]_{h}^{\alpha_{3}}$, for $h \in H, \alpha_{1}, \alpha_{2}, \alpha_{3} \in\{+,-, 0\}, x, y, z \in V$. An elementary membrane can be divided into two membranes with 
the same label, possibly transforming some objects and polarity.

These rules are applied according to the following principles:

- All the rules are applied in parallel and in a maximal manner. In one step, one object of a membrane can be used by only one rule (chosen in a nondeterministic way), but any object which can evolve by one rule of any form, should evolve.

- If a membrane is dissolved, then its content (multiset and internal membranes) is left free in the surrounding region.

- All objects and membranes not specified in a rule and which do not evolve remain unchanged to the next step.

- If at the same time a membrane $h$ is divided by a rule of type (e) and there are objects in this membrane which evolve by means of rules of type (a), then we suppose that first the evolution rules of type (a) are used, and then the division is produced. Of course, this process takes only one step.

- The rules associated with membranes labelled with $h$ are used for all copies of this membrane. At one step, a membrane labelled with $h$ can be the subject of only one rule of types (b)-(e).

In order to give a formal representation in Prolog of the basic structures of $\mathrm{P}$ systems with active membranes using 2-division, the following representation will be considered.

\subsection{Representation of membrane structures}

A given configuration will be expressed by means of a labelled tree, where:

1. $\quad<>$ is the position to denote the root of the tree and it will be associated to the skin;

2. if $\left\langle i_{1}, \ldots, i_{n}\right\rangle$ is the position of a membrane $h$, then $\left\langle i, i_{1}, \ldots, i_{n}\right\rangle$ will denote the position of the $i$-th internal membrane to $h$.

There exists one difference between the above representation and the one we use in Prolog: in our Prolog representation, if in one step of the computation a membrane with label $\left\langle i_{1}, \ldots, i_{n}\right\rangle$ has $k$ internal membranes, then its children do not have to be labelled with $<1, i_{1}, \ldots, i_{n}>,<2, i_{1}, \ldots, i_{n}>, \ldots,<k-1$, $i_{1}, \ldots, i_{n}>,<k, i_{1}, \ldots, i_{n}>$.

This is because if one child membrane is dissolved, the other ones are not re- 
labelled. Besides, new membranes obtained by division are labelled with new indexes, not by filling the holes of previously dissolved membranes.

\subsection{Representation of configurations}

Let us remember that to give a configuration for a $\mathrm{P}$ system with active membranes consists in making explicit the membrane structure and the content of every membrane present in this structure.

In our model we will represent the configuration in one step of the evolution as a set of one-literal clauses, each of them representing each alive membrane. Hence, in this representation each clause will show the label, position, polarity, multiset of objects and current step of the computation, as well as the $\mathrm{P}$ system this membrane belongs to. In this way, the set of clauses gives information about the contents of the membranes and the membrane structure (by means of the position of each one).

In a general form, to denote that in the $t$-th step of its evolution the $\mathrm{P}$ system, $P$, has a membrane at position [pos] with label $h$, polarity $\alpha$ and $m$ as multiset, we will write

$$
P:: \quad h \text { ec } \alpha \text { at }[p o s] \text { with } m \text { at_time } t
$$

Note that we can use the user-friendly representation of a Prolog literal, instead of the functional representation.

In a general way, if $m=\left\{\left\{x_{1}, \ldots, x_{n}\right\}\right\}$ (with not necessarily $x_{i} \neq x_{j}$ ), then we will denote $m=\left[x_{1}, \ldots, x_{n}\right]$.

\subsection{Description of the rules}

By means of some new function symbols, the rules are also represented as literals. In what follows we present the general form of the different rules showed above:

(a) $\left[{ }_{h} x \rightarrow u\right]_{h}^{\alpha}$

$P$ rule $x$ evolves_to $[u]$ in $h$ ec $\alpha$

(b) $x[h]_{h}^{\alpha_{1}} \rightarrow\left[{ }_{h} y\right]_{h}^{\alpha_{2}}$

$P$ rule $x$ out_of $h$ ec $\alpha_{1}$ sends_in $y$ of $h$ ec $\alpha_{2}$

(c) $\left[{ }_{h} x\right]_{h}^{\alpha_{1}} \rightarrow\left[{ }_{h}\right]_{h}^{\alpha_{2}} y$

$P$ rule $x$ inside_of $h$ ec $\alpha_{1}$ sends_out $y$ of $h$ ec $\alpha_{2}$

(d) $\left[{ }_{h} x\right]_{h}^{\alpha} \rightarrow y$

$P$ rule $x$ inside_of $h$ ec $\alpha$ dissolves_and_sends_out $y$ 
(e) $\left[{ }_{h} x\right]_{h}^{\alpha_{1}} \rightarrow\left[{ }_{h} y\right]_{h}^{\alpha_{2}}\left[{ }_{h} z\right]_{h}^{\alpha_{3}}$

$P$ rule $x$ inside_of $h$ ec $\alpha_{1}$ divides_into $y$ inside_of $h$

ec $\alpha_{2}$ and $z$ inside_of $h$ ec $\alpha_{3}$

\section{$\S 4$ The algorithm}

The Prolog algorithm to simulate a $\mathrm{P}$ system works in a natural way. The input of the program is the initial configuration of the membranes (which is represented as a set of literals with predicate symbol **, all of them at_time $0)$ and an appropriate set of rules.

- Step 1: Initialization. At the beginning, all the membranes are set to applicable and their objects are split into two multiset: one usable multiset, containing all the objects of the initial membrane, and one used multiset which is empty.

- Step 2: Transition. If there exists an applicable membrane satisfying the condition of a rule, then the rule is applied in the following way:

- (a)-step: At this stage, only rules of type (a) are checked. The object which triggers the rule is removed from the usable and the result multiset by the application of the rule is added to used, to prevent that the same object is used by two different rules at the same step. After the evolution step, the membrane remains to be applicable and new evolution rules can be applied.

This stage ends when no more rules of type (a) can be applied.

- Non-(a)-step: At this stage, only one rule of the other types (not (a)) can be applied. Let us remember that this simulation only works with deterministic P systems (in fact, it works with confluent ones). The action depends on the kind of rule to apply:

* Send out rule: The element which triggers the rule is removed from usable multiset and the new one is added to the used multiset of the father membrane. Both membranes changes to not applicable mode. If the element is sent out of the skin, then it is marked with the property outside.

* Send in rule: It is the reciprocal of the previous one. The element which triggers the rule is removed from usable in the father membrane and the new one is added to the used multiset. Both membranes changes to not applicable mode.

* Dissolution rule: The element which triggers the rule belongs to 
usable and the new element obtained together with the rest of the elements of the membrane are added to the used multiset of the father membrane. When a membrane $m$ is dissolved, its inner membranes (i.e. its children) become children of the father membrane of $m$ in the next stage of evolution. For that, the new positions have to be arranged. The father membrane changes to not applicable mode.

* Division rule: The element which triggers the rule belongs to usable and the division creates two new membranes in not applicable mode. One of them keeps the original position and the second one gets a position which has not been occupied by any membrane.

- End: When no more rules can be applied to applicable membranes, a new configuration (with at_time incremented by 1) is stored. In this moment no membrane has applicable or not applicable state. These modes only have validity during the evolution. At this stage, the $\mathrm{P}$ system is ready for a new step of the evolution.

- Step 3: End of computation. The evolution of the P system finishes when there are no rules to be applied.

Notice that due to the features of the implementation, the program only ensures a correct simulation of the evolution for deterministic (confluent) $\mathrm{P}$ systems. Nevertheless, most of the usual algorithms that solve interesting problems are covered.

\section{$\S 5$ A study case: SAT problem}

\subsection{A linear solution to the SAT problem by $\mathrm{P}$ systems with active membranes}

Propositional Satisfiability is the problem of determining, for a formula of the propositional calculus, if there is an assignment of truth values to its variables for which that formula evaluates to true. By SAT we mean the problem of propositional satisfiability for formulas in conjunctive normal form (CNF).

Following ${ }^{10)}$ we present a family of recognizing $\mathrm{P}$ systems with active membranes using 2-division (see ${ }^{6}$, , section 7.2 ) solving the SAT problem in linear time.

Let us suppose that $\varphi=C_{1} \wedge \cdots \wedge C_{m}$ in $\mathrm{CNF}$ and $\operatorname{Var}(\varphi)=\left\{x_{1}, \ldots, x_{n}\right\}$. For each $(m, n) \in \mathbf{N}^{2}$ we consider the recognizing $\mathrm{P}$ system

$$
(\Pi(\langle m, n\rangle), \Sigma(m, n), i(m, n)),
$$


where $\Sigma(m, n)=\left\{x_{i, j}, \bar{x}_{i, j}: 1 \leq i \leq m, 1 \leq j \leq n\right\}, i(m, n)=2$ and

$$
\Pi(\langle n, m\rangle)=\left(\Gamma(m, n),\{1,2\},\left[{ }_{1}[2]_{2}\right]_{1}, w_{1}, w_{2}, R\right)
$$

is defined as follows:

$$
\begin{aligned}
\Gamma(m, n) & =\Sigma(m, n) \cup\left\{c_{k}: 1 \leq k \leq m+2\right\} \cup\left\{d_{k}: 1 \leq k \leq 3 n+2 m+3\right\} \\
& \cup\left\{r_{i, k}: 0 \leq i \leq m, 1 \leq k \leq 2 n\right\} \cup\{e, t\} \cup\{\text { Yes }, \text { No }\} .
\end{aligned}
$$

We will say that every internal membrane with label 2 is an internal membrane. The initial content of each membrane is: $w_{1}=\emptyset$ and $w_{2}=\left\{d_{1}\right\}$.

The set $R$ of rules is given by:

(a) $\quad\left\{\left[{ }_{2} d_{k}\right]_{2}^{0} \rightarrow\left[{ }_{2} d_{k}\right]_{2}^{+}\left[{ }_{2} d_{k}\right]_{2}^{-}: 1 \leq k \leq n\right\}$.

By using a rule of $(a)$, a membrane with label 2 is divided into two membranes with the same label, but with different polarizations. These rules allow us to duplicate, in one step, the total number of internal membranes.

(b) $\left\{\left[{ }_{2} x_{i, 1} \rightarrow r_{i, 1}\right]_{2}^{+},\left[2 \bar{x}_{i, 1} \rightarrow r_{i, 1}\right]_{2}^{-}: 1 \leq i \leq m\right\}$, $\left\{\left[{ }_{2} x_{i, 1} \rightarrow \lambda\right]_{2}^{-}, \quad\left[2 \bar{x}_{i, 1} \rightarrow \lambda\right]_{2}^{+}: 1 \leq i \leq m\right\}$.

The rules of $(b)$ try to implement a process allowing to the internal membranes to encode the assignment of a variable and, simultaneously, to check the value of all clauses by this assignment, in such a way that if the clause is true, then an object $r_{i, 1}$ will appear in the membrane. In other case, the object encoding the variable will disappear.

(c) $\left\{\left[{ }_{2} x_{i, j} \rightarrow x_{i, j-1}\right]_{2}^{+},\left[{ }_{2} x_{i, j} \rightarrow x_{i, j-1}\right]_{2}^{-}: 1 \leq i \leq m, 2 \leq j \leq n\right\}$, $\left\{\left[2 \bar{x}_{i, j} \rightarrow \bar{x}_{i, j-1}\right]_{2}^{+},\left[2 \bar{x}_{i, j} \rightarrow \bar{x}_{i, j-1}\right]_{2}^{-}: 1 \leq i \leq m, 2 \leq j \leq n\right\}$.

The check process described previously is always made with respect to the first variable appearing in the internal membrane. Hence, the rules of $(c)$ take charge of making a cyclic path through all the variables to get that, initially, the first variable is $x_{1}$, then $x_{2}$, and so on.

(d) $\left\{\left[{ }_{2} d_{k}\right]_{2}^{+} \rightarrow\left[{ }_{2}\right]_{2}^{0} d_{k}, \quad,\left[{ }_{2} d_{k}\right]_{2}^{-} \rightarrow\left[{ }_{2}\right]_{2}^{0} d_{k}: 1 \leq k \leq n\right\}$, $\left\{d_{k}[2]_{2}^{0} \rightarrow\left[{ }_{2} d_{k+1}\right]_{2}^{0}: 1 \leq k \leq n-1\right\}$.

The rules of $(d)$ are used as controllers of the generating process of the assignments and the encoding of the satisfied clauses: the objects $d$ are sent out to the skin at the same time the checking is made and they come back to the internal membranes to start the division of these membranes.

(e) $\left.\quad\left\{{ }_{2} r_{i, k} \rightarrow r_{i, k+1}\right]_{2}^{0}: 1 \leq i \leq m, 1 \leq k \leq 2 n-1\right\}$. 
The use of objects $r$ in the rules $(i),(j)$ and $(k)$ makes necessary to perform a rotation of these objects. This is the mission of the rules of $(e)$.

(f) $\left\{\left[{ }_{1} d_{k} \rightarrow d_{k+1}\right]_{1}^{0}: n \leq k \leq 3 n-3\right\} ; \quad\left[{ }_{1} d_{3 n-2} \rightarrow d_{3 n-1} e_{0}\right]_{1}^{0}$.

Through the counter-objects $d$, the rules of $(f)$ control the rotation of the elements $r_{i, k}$ in the internal membranes.

(g) $\quad e\left[{ }_{2}\right]_{2}^{0} \rightarrow\left[{ }_{2} c_{1}\right]_{2}^{+} ; \quad\left[{ }_{1} d_{3 n-1} \rightarrow d_{3 n}\right]_{1}^{0}$.

The application of the rules of $(g)$ will show that the system is ready to check which clauses are made true by the assignment encoded by an internal membrane.

(h) $\left\{\left[{ }_{1} d_{k} \rightarrow d_{k+1}\right]_{1}^{0}: 3 n \leq k \leq 3 n+2 m+2\right\}$.

The rules of $(h)$ supply counters in the skin through objects $d$, in such a way that, if objects $d_{3 n+2 m}$ appear, then they show the end of the checking of the clauses. The objects $d_{k}$, with $3 n+2 m+1 \leq k \leq 3 n+2 m+3$, will control the final stage of the computation.

(i) $\left.\left[{ }_{2} r_{1,2 n}\right]_{2}^{+} \rightarrow\left[{ }_{2}\right]\right]_{2}^{-} r_{1,2 n}$.

The applicability of the rule $(i)$ encodes the fact that an internal membrane makes true the clause represented by the object $r_{1,2 n}$, through a change in the sign of its polarization. Because of this, we must relabel the values of $r$ representing the different internal membranes. This is done by means of the rules $(j)$.

(j) $\quad\left\{\left[{ }_{2} r_{i, 2 n} \rightarrow r_{i-1,2 n}\right]_{2}^{-}: 1 \leq i \leq m\right\}$.

(k) $r_{1,2 n}[2]_{2}^{-} \rightarrow\left[{ }_{2} r_{0,2 n}\right]_{2}^{+}$.

By using the rule $(k)$ the task of making explicit the assignments that make true the clause encoded in that moment of the execution by the object $r_{1,2 n}$ is ended.

(l) $\left\{\left[{ }_{2} c_{k} \rightarrow c_{k+1}\right]_{2}^{-}: 1 \leq k \leq m\right\}$.

The presence of objects $c_{k}$ (with $2 \leq k \leq m+1$ ) in the internal membranes shows that the assignments making true every clause are being determined.

(m) $\quad\left[{ }_{2} c_{m+1}\right]_{2}^{+} \rightarrow\left[{ }_{2}\right]_{2}^{+} c_{m+1}$. 
The rule $(m)$ sends to skin the objects $c_{m+1}$ appearing in the internal membranes.

(n) $\quad\left[{ }_{1} c_{m+1} \rightarrow c_{m+2} t\right]_{1}^{0}$.

By using the rule $(n)$ the objects $c_{m+1}$ in the skin evolve to objects $c_{m+2} t$. The objects $t$ in the skin are produced simultaneously with the appearance of the objects $d_{3 n+2 m+2}$ in the skin, and will show that there exists some assignment making true the formula.

(o) $\left[{ }_{1} t\right]_{1}^{0} \rightarrow\left[{ }_{1}\right]_{1}^{+} t$.

The rule $(o)$ sends out of the system an object $t$ changing the polarization of the skin membrane to positive (after that, the objects $t$ remaining in the skin region can no longer evolve). Hence, an object $c_{m+2}$ can exit the skin producing an object $Y e s$. This object is then sent to the environment through the rule $(p)$, telling us that the formula is satisfiable, and the computation stops.

(p) $\left[{ }_{1} c_{m+2}\right]_{1}^{+} \rightarrow\left[{ }_{1}\right]_{1}^{-}$Yes.

The applicability of the rule $(p)$ changes the polarization of the skin membrane to negative in order that the objects $c_{m+2}$ remaining in it cannot continue evolving.

(q) $\left[{ }_{1} d_{3 n+2 m+3}\right]_{1}^{0} \rightarrow[1]_{1}^{+}$No.

By the rule $(q)$ the object $d_{3 n+2 m+3}$ only evolves when the skin has a neutral charge (this is the case when the formula is not satisfiable). Then the system will evolve sending to the environment an object $N o$ and changing the polarization of the skin to positive, in order that objects $d_{3 n+2 m+3}$ remaining in the skin region can no longer evolve.

In ${ }^{10)}$ it is proved that the family $\Pi=(\Pi(t))_{t \in \mathbf{N}^{+}}$solves the $\mathbf{S A T}$ problem in linear time.

As input data for this $\mathrm{P}$ system, associated with the formula $\varphi=C_{1} \wedge \cdots \wedge C_{m}$ in CNF with $\operatorname{Var}(\varphi)=\left\{x_{1}, \ldots, x_{n}\right\}$, we consider the multiset

$$
\left\{x_{i, j}: x_{j} \in C_{i}\right\} \cup\left\{\bar{x}_{i, j}: \neg x_{j} \in C_{i}\right\}
$$

The execution of the $\mathrm{P}$ system with the above input can be structured in four stages: a stage of generation of all assignments, a stage of synchronization, a stage of checking the assignments with regard to the formula, and a stage of output. 
The generating stage is controlled by the objects $d_{i}$, with $1 \leq i \leq n$.

- The presence in the skin of one object $d_{i}$, with $1 \leq i \leq n$, will show that all possible partial assignments associated with $\left\{x_{1}, \ldots, x_{i}\right\}$ have been generated.

- In this stage, simultaneously to the consideration of partial assignments (each one associated with each internal membrane created by division) we will encode in every internal membrane all the clauses being true by the assignment represented by the membrane (through the objects $r_{i, k}$ ).

- The object $d_{1}$ appears in the skin after the execution of 2 steps. From the appearance of $d_{i}$ in the skin to the appearance of $d_{i+1}$, with $1 \leq i \leq n-1$, 3 steps have been executed.

- This stage ends when the object $d_{n}$ appears in the skin.

Hence, the total number of steps in the generating stage is $3 n-1$.

The synchronization stage has the goal of unifying the second subindexes of the objects $r_{i, k}$, to make them equal to $2 n$.

- This stage starts with the evolution of the object $d_{n}$ in the skin region.

- In every step of this stage the object $d_{i}$, with $n \leq i \leq 3 n-1$, evolves to $d_{i+1}$ in the skin region.

- This stage ends as soon as the object $d_{3 n}$ appears in the skin, that is the moment when each internal membrane has positive charge and contains one object $c_{1}$ (obtained by using the first rule of $(g)$ ).

Therefore, the synchronization stage needs a total of $2 n$ steps.

The checking stage has the goal to determine how many (and which) clauses are true in every internal membrane (that is, by the assignment represented by it). This stage is controlled by the objects $c_{i}$, with $1 \leq i \leq m+1$, and it starts after the presence of $c_{1}$ in the internal membranes.

- The presence of an object $c_{i}$ in an internal membrane shows that the clauses $C_{1}, \ldots, C_{i-1}$ are true by the assignment represented by that membrane.

- From every $c_{i}$ (with $1 \leq i \leq m$ ) the object $c_{i+1}$ is obtained in some membranes after the execution of 2 steps.

- The checking stage ends as soon as the object $d_{3 n+2 m}$ appears in the skin. Therefore, the total number of steps of this stage is $2 m$.

The output stage starts immediately after the appearance of the object $d_{3 n+2 m}$ in the skin and it is controlled by the objects $c_{m+1}$ and $c_{m+2}$.

- To produce the output $Y$ es the object $c_{m+1}$ must have been produced 
in some internal membrane of the configuration $\mathcal{C}_{5 n+2 m-1}$. Then, after 4 steps the system returns $Y$ es to the environment, through the evolution of objects $c_{m+2}$ present in the skin membrane, and when it has positive charge.

- To produce the output $N o$, no object $c_{m+1}$ appears in any internal membrane of the configuration $\mathcal{C}_{5 n+2 m-1}$. Then after 4 steps the system returns $N o$ to the environment, through the evolution of objects $d_{3 n+2 m+3}$ present in the skin region, and when it has neutral charge.

Therefore, the total number of steps in the output stage is 4 , and the total execution time of the $\mathrm{P}$ system is $5 n+2 m+3$.

\subsection{A Prolog session for $m=n=2$}

In this section we present a session for one instance of the problem. We consider two clauses: $C_{1}=x_{1} \wedge x_{2}$ and $C_{2}=x_{1} \wedge \neg x_{2}$. First, we will build the initial configuration and the set of rules ${ }^{* 2}$.

The initial configuration has two membranes: the skin at position $<>$, label $\mathrm{e}$, electrical charge 0 and empty content, and one internal membrane. The internal membrane contains the information relative to this instance of the problem: $x_{-} i_{-}$represents that $x_{j}$ is a variable in $C_{i}$ and $z i_{-} j$ represents that $\neg x_{j}$ occurs in $C_{j}$. So, we consider an internal membrane at position $<1>$ with label e2, electric charge 0 and the multiset codifying the information of the literals. We call $\mathrm{p} 1$ this $\mathrm{P}$ system and we write at_time 0 to denote that the system has not evolved yet.

The Prolog representation of this initial configuration is

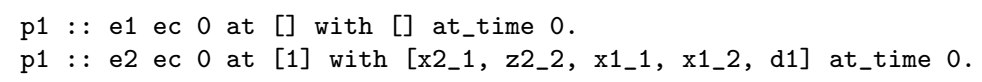

The set of rules only depends on the set of clauses and the set of variables. This set is generated by the simulator as a numbered set of rules. We have 52 rules in this example, and they can be found in the appendix.

To start with the simulation of the evolution of the $\mathrm{P}$ system $\mathrm{p} 1$ from time 0 we type the following command.

?- evolve $(\mathrm{p} 1,0)$.

The simulator returns the configuration at time 1 , the set of rules and additional information about the elements outside the skin membrane.

*2 The Prolog simulator provides the facilities to build automatically the initial configuration and the set of rules for any instance of the SAT problem. 


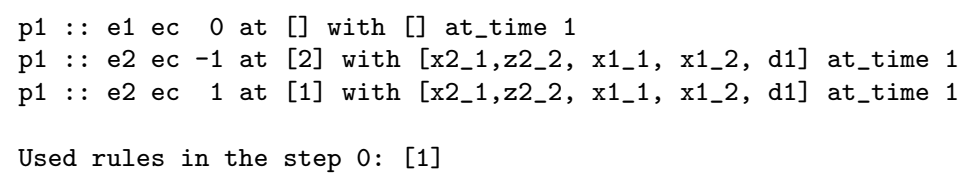

In this step only the first rule has been applied. We follow the evolution.

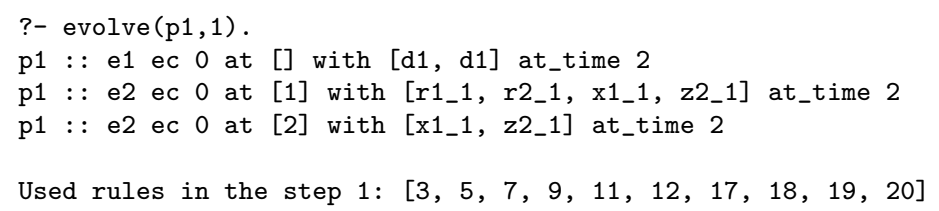

where 10 rules have been applied.

The simulator also allows us to go to a configuration at time $\mathrm{N}$ without showing the previous steps.

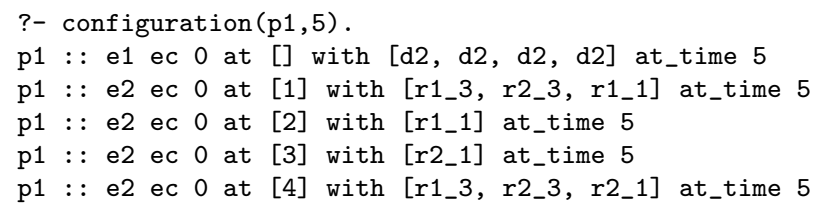

In this example the generating stage ends at time 5 (that is, $3 n-1$ ). We can follow the evolution and obtain the configuration at time 9 (that is, $3 n-1+2 n$ ). This is the first configuration where the element $d 6$ appears in the skin region.

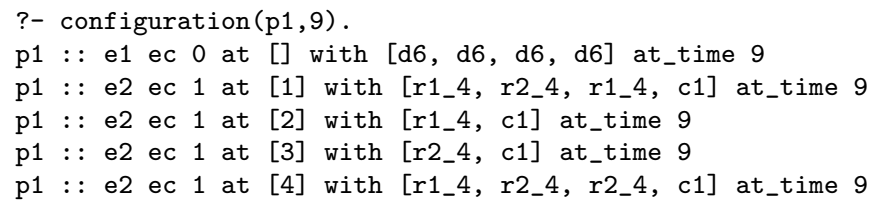

The synchronization stage has ended. In the next step the checking stage begins; it ends at time 13 (that is, $5 n-1+2 m$ ) when the element d10 appears in the skin region.

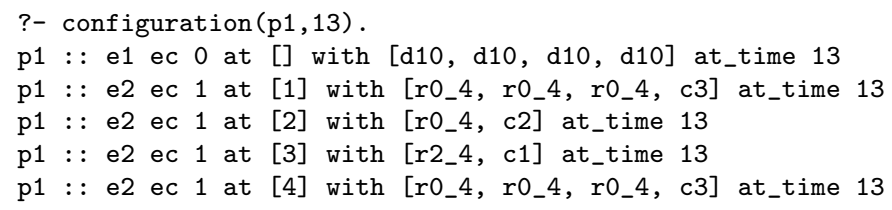

After that, the output stage starts. At step 16 (that is, $5 n+2 m+2$ ) the element $t$ is sent out of the skin region. 


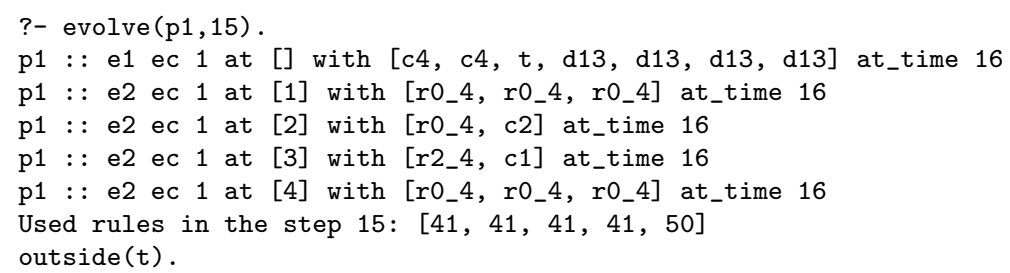

Finally, in the next step the system sends out yes

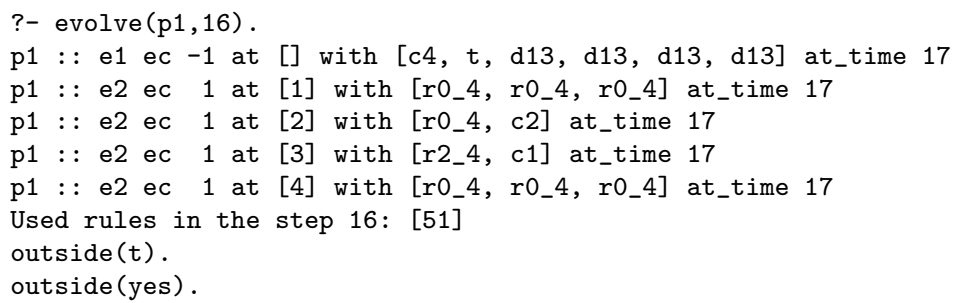

To check the system we try to evolve one more time, though this is a halting configuration.

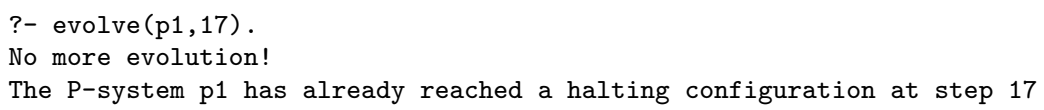

\section{$\S 6$ Conclusion and future work}

We have presented an effective tool for making experiments with $\mathrm{P}$ systems to solve decision problems. One example is the propositional satisfiability problem, but the simulator is useful for general purposes, covering all the deterministic P systems with active membranes using 2-division.

In the literature some references to other software simulators can be found (e.g. ${ }^{1,2,4,11)}$ ), but this is the first time when $\mathrm{P}$ systems with active membranes are simulated. The automatic generation of the initial configuration and the set of rules by the system from the formal description of the problem is other relevant feature of the simulator presented in this paper.

The chosen logic representation is so user-friendly that the user can follow the evolution of the system without any knowledge about Prolog language. Besides, representing rules and membranes as literals in a clausal language allows a natural treatment of the objects from a programmer's point of view.

The idea of representing the $\mathrm{P}$ systems as an unordered set of clauses endows them with a flexibility which can be used for new purposes. This can be 
a good starting point towards new unexplored perspectives in $\mathrm{P}$ systems, such as the merge of two $\mathrm{P}$ systems or the evolution of a population of $\mathrm{P}$ systems in the way of genetic algorithms.

Representing P systems as Prolog programs suggests new relations between $\mathrm{P}$ systems and other deeply studied techniques in Artificial Intelligence. Different aspects related to heuristics, search in spaces of states or automated learning can be linked to the theory of $\mathrm{P}$ systems via this representation.

\section{Acknowledgment}

The last two authors gratefully acknowledge the support of this research through the project TIC2002-04220-C03-01 of the Ministerio de Ciencia y Tecnología de España, cofinanced by FEDER funds.

\section{References}

1) Arroyo, F.; Luengo, C.; Baranda, A.V.; de Mingo, L.F.: A software simulation of transition P systems in Haskell, Springer-Verlag, LNCS 2597, Berlin, 2003, 19-32.

2) Balbontín Noval, D.; Pérez Jiménez, M.J.; Sancho Caparrini, F.: A MzScheme implementation of transition P systems, Springer-Verlag, LNCS 2597, Berlin, 2003, 58-73.

3) Bratko, I.: PROLOG Programming for Artificial Intelligence, Third edition. Addison-Wesley, 2001.

4) Ciobanu, G.; Paraschiv, D.: Membrane Software. A P System Simulator, Fundamenta Informaticae 49 , 1-3 (2002), 61-66.

5) Păun, G.: Computing with membranes, Journal of Computer and System Sciences, 61(1), 2000, 108-143.

6) Păun, G.: Membrane Computing. An Introduction, Springer-Verlag, Berlin, 2002.

7) Păun, G.; Rozenberg, G.: A guide to membrane computing, Theoretical Computer Sciences, 287, 2002, 73-100.

8) Păun, G.; Rozenberg, G.; Salomaa, A.: Membrane computing with external output, Fundamenta Informaticae, 41(3), 2000, 313-340.

9) Pérez Jiménez, M.J.; Romero-Jiménez, A.; Sancho-Caparrini, F.: Teoría de la Complejidad en modelos de computación celular con membranas, Editorial Kronos, Sevilla, 2002.

10) Pérez-Jiménez, M.J.; Romero-Jiménez, A.; Sancho-Caparrini, F: The polynomial complexity class in $\mathrm{P}$ systems using membrane division. Submitted.

11) Suzuki, Y.; Tanaka, H.: On a LISP Implementation of a Class of P Systems, Romanian J. of Information Science and Technology, 3, 2 (2000), 173-186.

12) The P Systems Web Page: http://psystems.disco.unimib.it/ 
13) Logic Programming: http://www.afm.sbu.ac.uk/logic-prog/

\section{$\S 7 \quad$ Appendix}

In what follows we present a sample of the rules written in the format they must be given to the simulator*3.

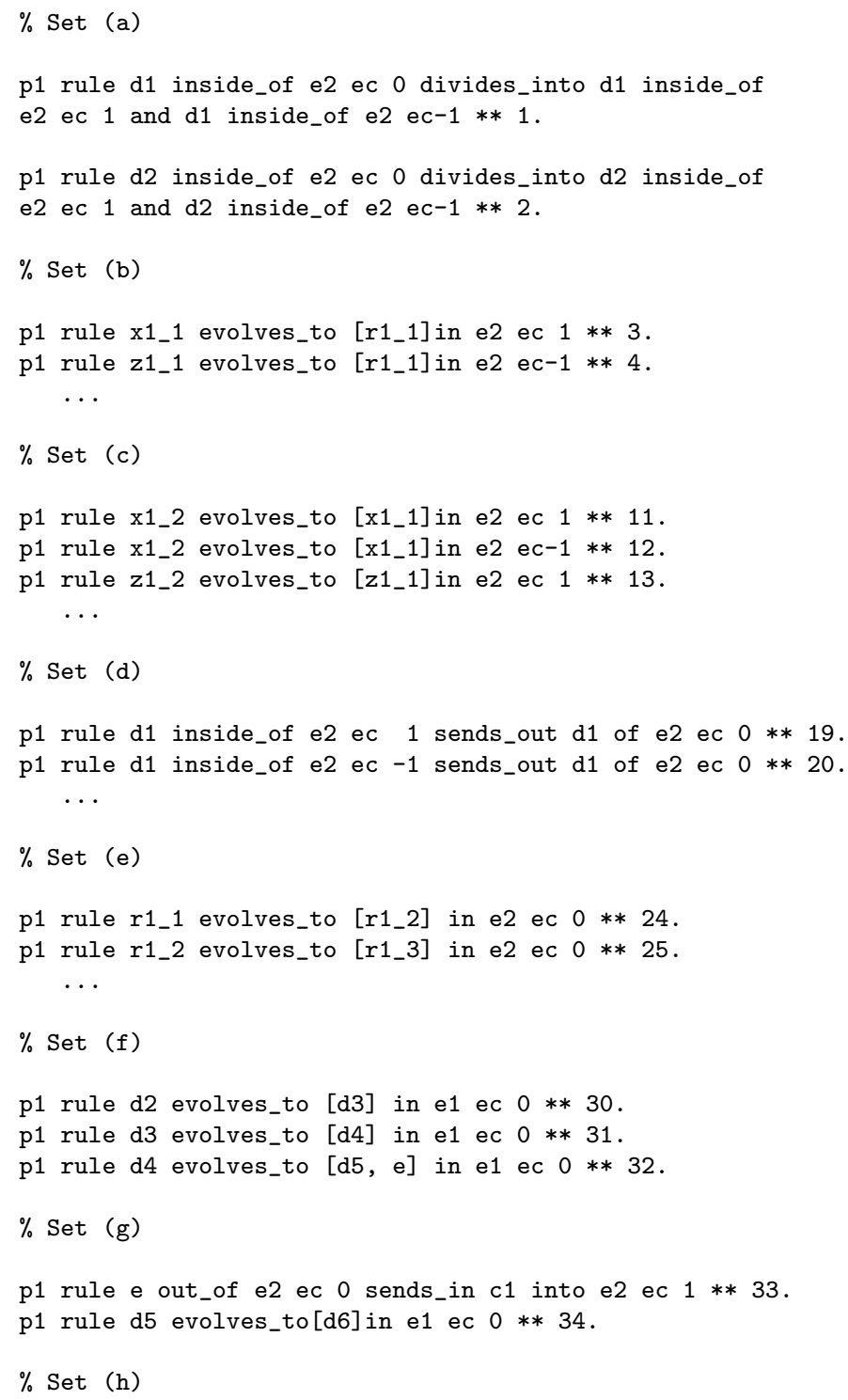

*3 The number after ** is the ordinal associated to the rule. 
p1 rule d6 evolves_to [d7] in e1 ec $0 * * 35$.

...

p1 rule d12 evolves_to [d13] in e1 ec $0 * * 41$.

$\% \operatorname{Set}(i)$

p1 rule r1_4 inside_of e2 ec 1 sends_out $r 1 \_4$ of e2 ec-1 ** 42 .

$\% \operatorname{Set}(j)$

p1 rule r1_4 evolves_to [r0_4] in e2 ec $-1 * * 43$.

p1 rule r2_4 evolves_to [r1_4] in e2 ec $-1 * * 44$.

$\% \operatorname{Set}(\mathrm{k})$

p1 rule r1_4 out_of e2 ec-1 sends_in r0_4 into e2 ec $1 * * 45$.

$\% \operatorname{Set}(1)$

p1 rule c1 evolves_to [c2] in e2 ec $-1 * * 46$.

p1 rule c2 evolves_to [c3] in e2 ec $-1 * * 47$.

$\%$ Set (m)

p1 rule c3 inside_of e2 ec 1 sends_out c3 of e2 ec $1 * * 48$.

$\% \operatorname{Set}(n)$

p1 rule c3 evolves_to $[c 4, t]$ in e1 ec $0 * * 49$.

$\% \operatorname{Set}(0)$

p1 rule $t$ inside_of e1 ec 0 sends_out $t$ of e1 ec $1 * * 50$.

$\% \operatorname{Set}(p)$

p1 rule c4 inside_of e1 ec 1 sends_out yes of e1 ec-1 ** 51 .

$\% \operatorname{Set}(q)$

p1 rule d13 inside_of e 1 ec 0 sends_out no of e1 ec $1 * * 52$. 